\title{
Morphology, polarity, and lateral molecular beam epitaxy growth of GaN on sapphire
}

\author{
E. C. Piquette, ${ }^{\text {a) }}$ P. M. Bridger, Z. Z. Bandić, and T. C. McGill \\ Thomas J. Watson, Sr. Laboratory of Applied Physics, California Institute of Technology, Pasadena, \\ California 91125
}

(Received 5 October 1998; accepted 31 January 1999)

\begin{abstract}
Gallium nitride was grown on sapphire (0001) substrates by radio frequency plasma assisted molecular beam epitaxy. The surface morphology was characterized during growth by reflection high energy electron diffraction, and ex situ by scanning electron microscopy (SEM), atomic force microscopy (AFM) and x-ray diffraction. It is found that surface morphological features are linked to domains of specific wurtzite crystal polarity, $(0001) \mathrm{Ga}$ face or $(000 \overline{1}) \mathrm{N}$ face, for Ga-rich growth. For growth on AlN buffer layers, we commonly observe films which consist of largely (0001)Ga polarity material, as confirmed by selective etch tests, with a varying coverage of $(000 \overline{1}) \mathrm{N}$-face inversion domains threading along the growth direction. For growth near stoichiometric conditions, the growth rate of the $\mathrm{N}$-face domains is slightly lower than that for the Ga-face matrix, which results in the formation of pits with inversion domains at their centers. For samples grown by first depositing GaN under N-rich conditions, followed by growth under Ga-rich conditions, a different morphology is obtained, exhibiting large hexagonal flat terraces observable by SEM and AFM. The apparent grain size of these films is increased substantially over films grown using a single step approach. The cross sectional SEM images of the two-step films show a network of voids and columns at the interface between the N-rich and the Ga-rich layers, above which micron-scale islands form and coalesce via lateral growth. Lateral growth may result in reduced defect density and improved crystal quality. The asymmetric x-ray peak ( $11 \overline{2} 4)$ width is reduced to approximately 280 arcsec in the two-stage GaN films. (C) 1999 American Vacuum Society.

[S0734-211X(99)02303-3]
\end{abstract}

\section{INTRODUCTION}

Gallium nitride is becoming established as a versatile material for wideband gap semiconductor devices. Recently there has been great interest in $\mathrm{GaN}$, InGaN, and $\mathrm{AlGaN}$ based devices, not only for optoelectronics, ${ }^{1}$ but also for high power, ${ }^{2,3}$ high frequency, ${ }^{4}$ and high temperature applications. For improved device performance in all these applications, it is essential to produce materials of high crystal quality, with low defect density, high carrier mobility, and with flat surfaces. These qualities in $\mathrm{GaN}$ films continue to improve, despite the lack of suitable GaN substrates, using techniques such as metalorganic vapor phase epitaxy (MOVPE), hydride vapor phase epitaxy (HVPE), and molecular beam epitaxy (MBE). Despite the vast amount of research and the considerable improvement in material quality that has been achieved, it remains clear that we are still far from fully understanding all the details and mechanisms of the growth of GaN, particularly by MBE.

Many difficulties arise in the growth of GaN epitaxial layers because of the use of a highly mismatched substrate, such as sapphire or silicon carbide. Silicon carbide has many advantages over sapphire for GaN growth, including smaller mismatch in lattice parameter and a distinct crystal polarity, but $c$-plane sapphire is more often used for reasons of cost. In order to produce the highest quality $\mathrm{GaN}$ films on sap-

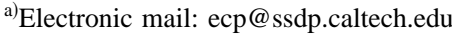

phire, great care must be given to the conditions of film nucleation and buffer layer deposition, so as to initiate the growth of films of the desired crystal polarity ${ }^{5,6}$ and that are free of inversion (anti-phase) domains (IDs). ${ }^{7}$ In MOVPE deposited films, growing on the $(000 \overline{1}) \mathrm{N}$ face of GaN has resulted in extremely rough surfaces with large pyramidal features, while growing on the (preferred) (0001)Ga face can result in smooth surfaces and overall higher quality films. ${ }^{8}$ Dissimilarly, for MBE growth using a nitrogen plasma source, most reports have been of mixed polarity films ${ }^{9}$ or films of $(000 \overline{1}) \mathrm{N}$-face polarity. ${ }^{10}$ Only recently have there been confirmations of Ga-face growth by rf MBE using AlN buffer layers. ${ }^{6,11}$ Locally flat surfaces can be achieved on both polarities by MBE under slightly Ga-rich conditions, but special steps need to be taken to produce films that are free of inversion domains, and there are few reported procedures for doing so. ${ }^{12,13}$ Lateral overgrowth of inversion domains is routine in MOVPE films, where the Ga-face matrix overgrows $\mathrm{N}$-face columnar domains near the film-substrate interface, forming "house-like" structures that are observable by transmission electron microscopy (TEM). ${ }^{14,15}$ Here we report on the possibility of producing inversion-domainfree (homopolar) films by over growing the inversion domains under highly Ga-rich growth conditions. We demonstrate the lateral growth of $\mathrm{GaN}$ by $\mathrm{MBE}$ using a two-stage technique for (0001) Ga-face $\mathrm{GaN}$, producing films with in- 
creased grain size that are formed by coalescence of flattopped hexagonal islands.

\section{EXPERIMENT}

A series of GaN layers was grown on c-plane (0001) sapphire substrates by radio frequency plasma assisted molecular beam epitaxy (MBE). The substrates were backside coated with molybdenum or titanium and loaded into the vacuum system without chemical treatment. The radio frequency plasma source (SVT Associates) was operated at 350 $\mathrm{W}$ and a system pressure of $6-8 \times 10^{-5}$ Torr. The substrates were thermally cleaned prior to growth at $1000^{\circ} \mathrm{C}$ for 15 min after which they were exposed to the nitrogen radical beam until a bright, streaky reflection high energy electron diffraction (RHEED) pattern emerged. A nominally $25 \mathrm{~nm}$ thick AlN buffer layer was deposited on the nitrided surface at a substrate temperature of $600-650^{\circ} \mathrm{C}$, after which a twofold RHEED reconstruction was visible. GaN layers were grown on the AlN buffer layers under different flux conditions at a fixed temperature of $800^{\circ} \mathrm{C}$. A series of films was grown employing a $\mathrm{Ga} / \mathrm{N}$ flux ratio either $\mathrm{N}$ rich, $\mathrm{Ga}$ rich, or near 1:1. Additionally, some samples were grown using a two-step process, first depositing $\mathrm{GaN}$ under nitrogen-rich conditions, followed by growth under Ga-rich (approximately 2:1) fluxes. Films were characterized ex situ by atomic force microscopy (AFM) using a multi-mode Digital Instruments Nanoscope IIIA, and by scanning electron microscopy (SEM) using a JEOL 6400V. High-resolution x-ray diffraction (XRD) measurements were performed using a Philips diffractometer equipped with four-crystal Ge (440) collimation and a two-crystal Bonse-Hart triple axis detector. GaN polarity was determined by RHEED reconstruction at low temperature, ${ }^{10}$ and by molten $\mathrm{KOH}$ etching. ${ }^{8}$

\section{RESULTS AND DISCUSSION}

\section{A. Growth under stoichiometric and Ga-rich conditions}

The $\mathrm{Ga} / \mathrm{N}$ flux ratio drastically affects the surface properties in GaN growth by rf MBE. ${ }^{13,16,17}$ Growth under conditions of a slight excess of $\mathrm{Ga}$ is known to lead to locally flat GaN surfaces and streaky RHEED patterns while growth under Ga-deficient conditions results in spotty, faceted RHEED images and rough surfaces. Stoichiometric growth conditions are taken to be where the growth mode abruptly transforms from two dimensional to three dimensional. For samples grown near stoichiometric conditions, the RHEED pattern showed unreconstructed $(1 \times 1)$ streaks but sometimes displayed a small amount of faceting. Figure 1(A) shows the AFM surface scan of such a layer. We see that even though the RHEED image is streaky, the entire surface of the film is not flat. Pits are present on a mostly flat background. These pits are approximately $30 \mathrm{~nm}$ deep and $\sim 200 \mathrm{~nm}$ wide, and are believed to be induced by the presence of inversion domains. Myers et al. ${ }^{7}$ have observed similar effects for the case of Ga-polar inversion domains in a N-polar matrix, where the IDs formed hillocks. As has been shown by other
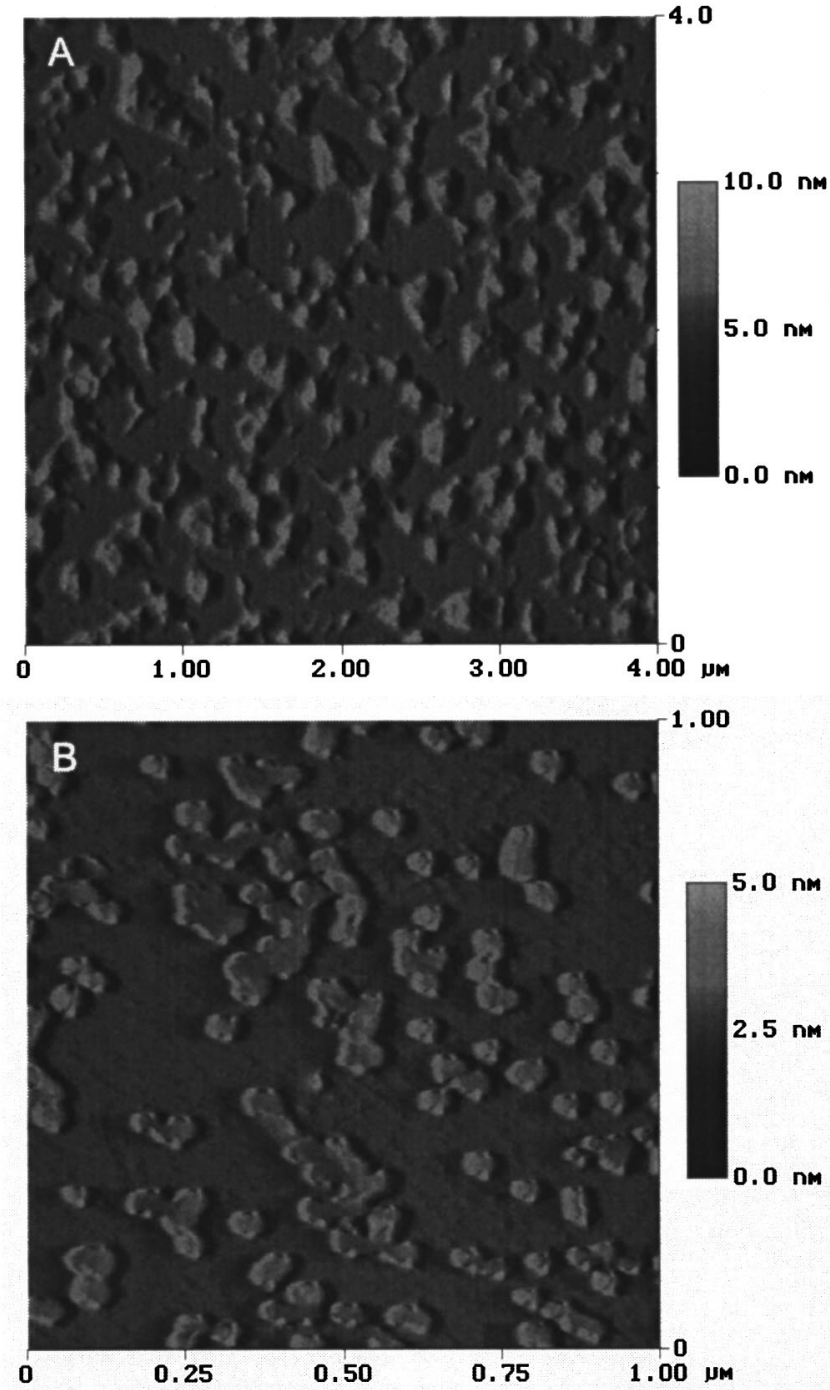

FIG. 1. AFM scans of the GaN surfaces for (A) a sample grown near stoichiometric flux conditions, and (B) a sample grown under Ga-rich conditions. The pits present on the flat background are thought to be caused by reduced growth rate at inversion domains. The images have been processed using the nanoscope high pass filter for clarity.

researchers,${ }^{18}$ by growing near stoichiometric conditions one can observe an apparent difference in growth rate between the $(0001) \mathrm{Ga}$ face and $(000 \overline{1}) \mathrm{N}$ faces of $\mathrm{GaN}$, as they are present in different domains of the film. For a (0001) film that contains inversion domains, we see that these $(000 \overline{1})$ domains thread along the growth direction at a lower growth rate than the surrounding material, inducing pits where they reach the surface. TEM measurements are needed to confirm this interpretation.

If the $\mathrm{Ga} / \mathrm{N}$ flux ratio is increased from $1: 1$, the growth rates of the two polarities tend to become equalized, and the depth of the pits is reduced. Very flat films can be grown under these slightly Ga-rich conditions. Figure 1(B) shows such a film, with pits that are approximately $2 \mathrm{~nm}$ deep and $40 \mathrm{~nm}$ wide. It should be noted that for high $\mathrm{Ga} / \mathrm{N}$ flux ratios, the growth temperature should be maintained high enough to 

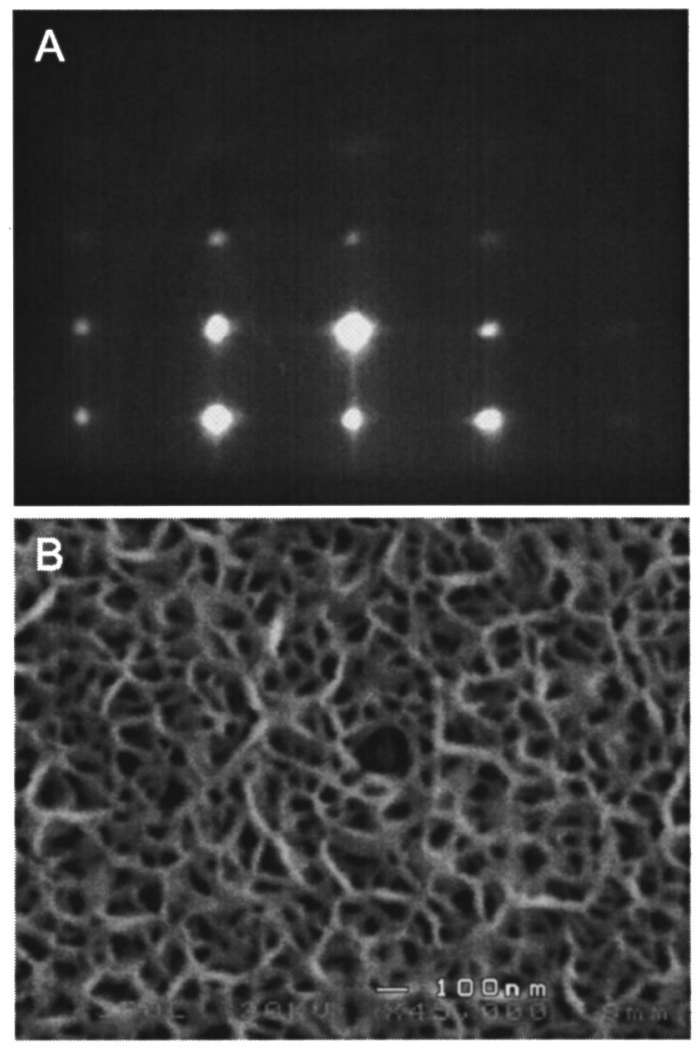

FIG. 2. (A) RHEED image of a GaN sample grown under highly N-rich flux conditions. Note the presence of both horizontal and vertical streaks superimposed on the spotty wurtzite transmission pattern. (B) The plan view SEM photograph of the same sample presents a complex mesh-like microstructure. This image was taken at a $20 \mathrm{kV}$ operating voltage and $45000 \times$ magnification. The pits have sidewalls that are predominantly $\{10 \overline{1} 0\}$ facets, and may correspond to opened threading dislocations.

re-evaporate excess $\mathrm{Ga}$ and thus avoid the formation of $\mathrm{Ga}$ droplets.

\section{B. Growth under N-rich conditions}

For $\mathrm{Ga} / \mathrm{N}$ flux ratios less than one, spotty and sometimes faceted RHEED patterns are observed during growth. It is plausible that the low flux ratio gives rise to a (partly) nitrogen terminated surface which has a high sticking coefficient for $\mathrm{Ga}$ atoms, but low surface mobility, and the morphology then evolves by statistical roughening. ${ }^{7}$ As the growth conditions become even more $\mathrm{N}$ rich, especially at higher growth temperatures, interesting and complicated morphological features can arise. The $\{11 \overline{2} 0\}$ azimuth RHEED pattern observed during growth for one such film is shown in Fig. 2(A). In general the spotty transmission pattern indicates a wurtzite film with a three dimensional surface. Some streaks can be seen overlaying this pattern both orthogonal and parallel to the sample surface plane. These streaks indicate faceting in the $\{0001\}$ and $\{10 \overline{1} 0\}$ planes, respectively. The vertical $\{10 \overline{1} 0\}$ facets (horizontal RHEED streaks) are unusual in the MBE growth of GaN, but indicate the high stability of these crystal surfaces. No horizontal streaks were observed for the $\{10 \overline{1} 0\}$ azimuthal RHEED image. The SEM plan view scan of a film grown with $\mathrm{Ga} / \mathrm{N}$ flux ratio of $1: 3$ is shown in Fig. 2(B). A mesh-like morphology is observed. These GaN fiber structures are highly oriented along the $\{11 \overline{2} 0\}$ crystalline directions, with some degree of misalignment. Equivalently, the sidewalls of the pits which lie between the fibers are aligned in these directions. The SEM data therefore also indicate the presence of $\{10 \overline{1} 0\}$ facets. The net growth rate of films under these conditions is slow, and the decomposition rate is high. The appearance of facets on the $\{10 \overline{1} 0\}$ and $\{0001\}$ planes suggests that the decomposition rate is slow on these planes. This variation in growth and decomposition rates for the different crystal directions and for high $\mathrm{N} / \mathrm{Ga}$ ratios is quite similar to results obtained in lateral epitaxial overgrowth of GaN by $\mathrm{MOVPE}^{19}$ and HVPE, and also TEM observations of nano pipes and pits. ${ }^{14}$ The morphology seen here can also be explained by the "opening up" of threading dislocations into nano pits in the highly nitrogen-rich reactive environment. The density of pits in Fig. 2(B) is approximately $10^{10} \mathrm{~cm}^{-2}$, which coincides with the density of extended defects in a typical MBE $\mathrm{GaN}$ film. This morphology also bears similarity to that reported for $\mathrm{GaN}$ annealed in forming gas. ${ }^{6}$

\section{Two-stage growth}

Some samples were grown by first depositing GaN under nitrogen-rich conditions, followed by growth under Ga-rich (approximately 2:1) fluxes. As above, the RHEED pattern during the first growth stage was spotty with faceting. We expect this layer to be highly defected and heteropolar. At the transition from N-rich growth to Ga-rich growth, the RHEED pattern initially became very dim, before evolving into an unreconstructed streaky pattern, and continued to improve with growth. Samples were removed after approximately $1 \mu \mathrm{m}$ (sample A) and $2 \mu \mathrm{m}$ (sample B) of Ga-rich growth. The surfaces of these films exhibit large hexagonal flat-topped mesas observable by SEM. SEM photographs of these surfaces are shown in Fig. 3. AFM micrographs ${ }^{20}$ are essentially similar and show no pits on the mesa structures. After $1 \mu \mathrm{m}$ of Ga-rich growth, the mesa structures of sample A remain mostly isolated. But after an additional micron of growth, we see that these mesas have started to coalesce [Fig. 3(C)]. At this point, the RHEED pattern is very streaky, showing high contrast $(1 \times 1)$ during growth and $(2 \times 1)$ reconstruction under nitrogen soak. During cooldown, clear $(2 \times 2)$ reconstructions can be observed below $750^{\circ} \mathrm{C}$. Figure 4 shows the cross sectional SEM image of sample B, in a region where several hexagonal islands have merged. It is apparent that columns grow up from the first, N-rich GaN layer, which then grow laterally and coalesce. We speculate that the growth under $\mathrm{N}$-rich conditions exploits the difference in growth rates between the (0001) and (0001) GaN directions, allowing Ga-face columnar domains to outgrow the $\mathrm{N}$-face regions. Upon commencement of the second layer, lateral growth is facilitated and the Ga-face protrusions overgrow the material of opposite polarity. The highly Garich conditions lead to droplet condensation at the edges of 

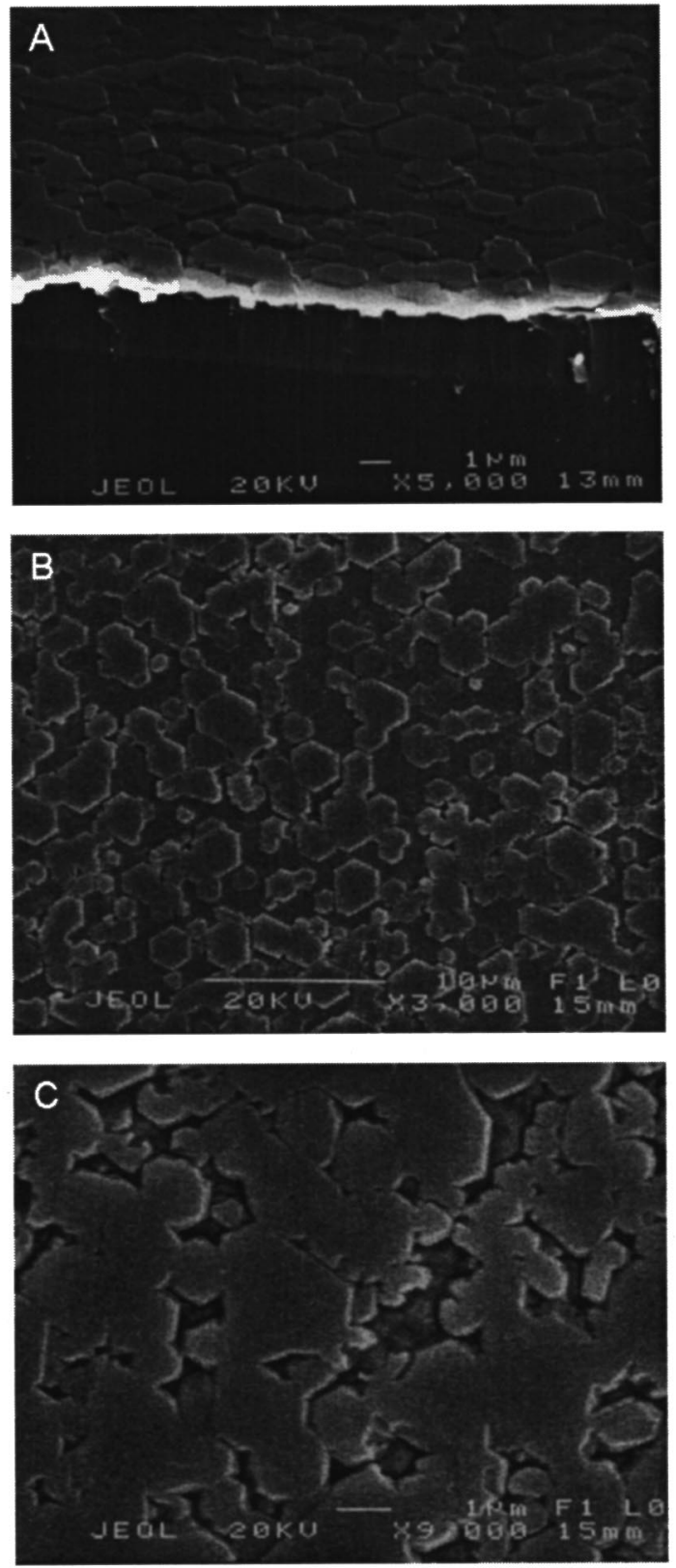

FIG. 3. SEM images of GaN samples grown first under N-rich conditions, followed by growth under Ga-rich conditions. (A) (oblique view) and (B) (plan view) show the formation of micron-size flat-topped hexagonal islands for two-stage sample A. After $1 \mu \mathrm{m}$ of growth, these islands are still mostly isolated. (C) SEM plan view of sample B after $2 \mu \mathrm{m}$ of growth under Ga-rich conditions. The hexagonal islands are now beginning to coalesce.

the growing islands, and ultimately form the voids that are present at the interface. This may be similar to the Ga-rich buffer layer technique of Refs. 13 and 21 or to results obtained by Held et al. ${ }^{12}$ where the formation of inversion domains could be suppressed by utilizing nucleation layers formed by repeatedly nitriding a condensed Ga surface on sapphire. Lateral growth of GaN may lead to a possible reduction in defect density and improved crystal quality, as occurs in MOVPE. ${ }^{19,22}$ TEM or other techniques have not yet been used to count the defects in the two-stage samples.
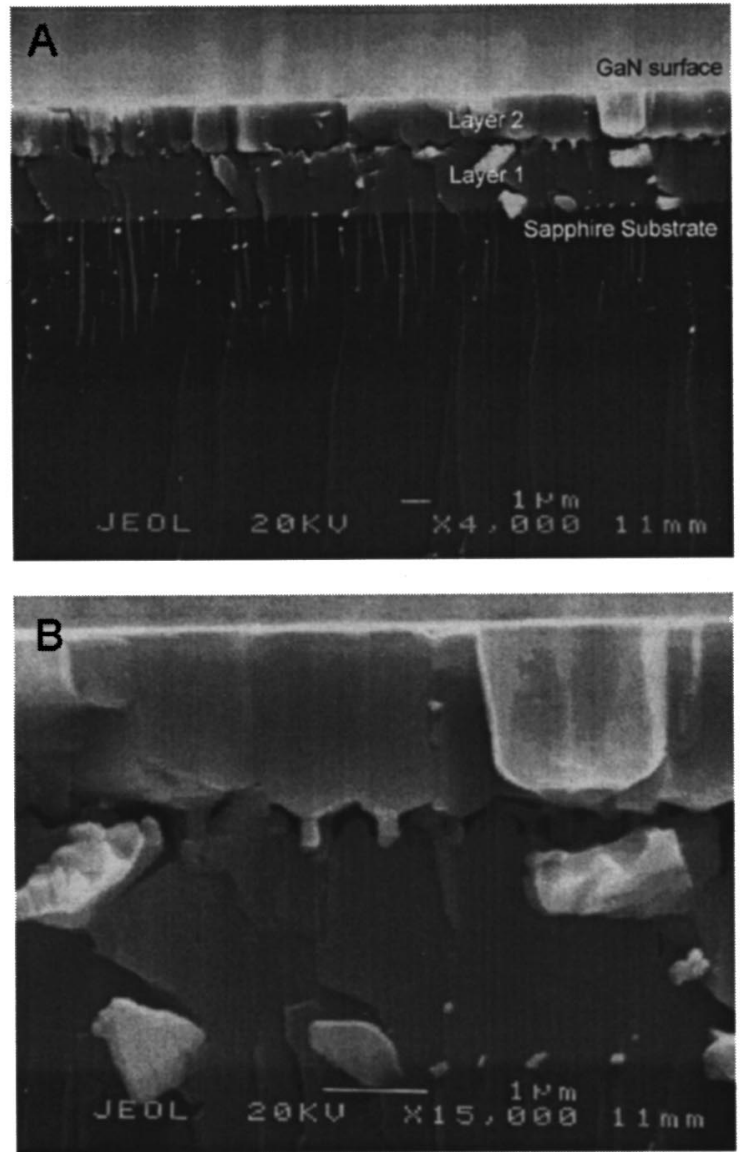

FIG. 4. (A) Cross sectional SEM view of sample B. Note the network of voids and columns at the interface between layer one, grown under $\mathrm{N}$-rich conditions, and layer two, grown under Ga-rich conditions. The presence of voids can be attributed to Ga condensation. It is evident that lateral growth of micron-scale islands proceeds in layer two. (B) Close-up of (A).

We have, however, observed a considerable improvement in structural quality as measured by $\mathrm{x}$-ray diffraction. Indeed, the asymmetric $\mathrm{x}$-ray peak $(11 \overline{2} 4)$ full width at half maximum (FWHM) is reduced from greater than $1000 \operatorname{arcsec}$ to as low as 280 arcsec in the two-stage GaN films. Some other traditional characterization techniques, such as Hall and photoluminescence, are made difficult in these samples because of the buried interface.

\section{SUMMARY}

Gallium nitride was grown on sapphire (0001) substrates by rf-MBE. The surface morphology was characterized by RHEED, SEM, and AFM. It was found that generally flat films can be grown using slightly Ga-rich flux conditions, but some contain pits indicating the presence of inversion domains. The growth and decomposition rates for various crystal directions are seen to be dependent on the flux conditions. Specifically, under N-rich conditions the $\{11 \overline{2} 0\}$ decomposition rates are higher than for the $\{10 \overline{1} 0\}$ and $\{0001\}$ directions, which can form stable facets. This is similar to growth by MOVPE. Near stoichiometric flux conditions, the (0001) growth rate is slightly higher than the $(000 \overline{1})$ growth 
rate. As growth becomes more Ga rich, the (0001) and $(000 \overline{1})$ growth rates become more equal, up to the point of Ga condensation, where droplets may form. For samples grown by first depositing $\mathrm{GaN}$ under N-rich conditions, followed by growth under Ga-rich conditions, lateral growth and coalescence of micron-scale islands can occur over an interface of networked columns and voids, leading to structurally superior surface layers.

\section{ACKNOWLEDGMENTS}

This work was supported by the Defense Advanced Research Project Agency, and monitored by Dr. Y. S. Park and the Office of Naval Research under Grant No. N00014-92-J1845.

${ }^{1}$ S. Nakamura and G. Fasol, The Blue Laser Diode (Springer, Berlin, 1997).

${ }^{2}$ Z. Z. Bandić, E. C. Piquette, P. M. Bridger, T. F. Kuech, and T. C. McGill, Mater. Res. Soc. Symp. Proc. 483, 399 (1998).

${ }^{3}$ Z. Z. Bandić, P. M. Bridger, E. C. Piquette, R. A. Beach, T. F. Kuech, and T. C. McGill, Solid-State Electron. 42, 2289 (1998).

${ }^{4}$ L. F. Eastman, K. Chu, J. Smart, and J. R. Shealy, Mater. Res. Soc. Symp. Proc. 512, 3 (1998).

${ }^{5}$ J. L. Rouviere, J. L. Weyher, M. Seelmann-Eggebert, and S. Porowski, Appl. Phys. Lett. 73, 668 (1998).

${ }^{6}$ E. S. Hellman, MRS Internet J. Nitride Semicond. Res. 3, 11 (1998).

${ }^{7}$ T. H. Myers, L. S. Hirsch, L. T. Romano, and M. R. Richards-Babb, J. Vac. Sci. Technol. B 16, 2261 (1998).
${ }^{8}$ M. Seelmann-Eggebert, J. L. Weyher, H. Obloh, H. Zimmermann, A. Rar, and S. Porowski, Appl. Phys. Lett. 71, 2635 (1997).

${ }^{9}$ L. T. Romano, B. S. Kruson, R. Singh, and T. D. Moustakas, J. Electron. Mater. 26, 285 (1997).

${ }^{10}$ A. R. Smith, R. M. Feenstra, D. W. Greve, M.-S. Shin, M. Skowronski, J. Neugebauer, and J. E. Northrup, Appl. Phys. Lett. 72, 2114 (1998).

${ }^{11}$ M. J. Murphy, T. J. Eustis, H. Wu, W. Yeo, W. J. Schaff, O. Ambacher, J. Smart, J. R. Shealy, and L. F. Eastman, presented at the Seventeenth North American Molecular Beam Epitaxy Conference, Penn State University, G.5, 1998.

${ }^{12}$ R. Held, D. E. Crawford, A. M. Johnson, A. M. Dabiran, and P. I. Cohen, Surf. Rev. Lett. 5, 913 (1998).

${ }^{13}$ S. L. Buczkowski, Z. Yu, M. Richards-Babb, N. C. Giles, L. T. Romano, and T. H. Myers, Mater. Res. Soc. Symp. Proc. 449, 197 (1997).

${ }^{14}$ J. L. Rouviere, M. Arlery, R. Niebuhr, K. H. Bachem, and O. Briot, Mater. Sci. Eng., B 43, 161 (1997).

${ }^{15}$ X. H. Wu, L. M. Brown, D. Kapolnek, S. Keller, B. Keller, S. P. DenBaars, and J. S. Speck, J. Appl. Phys. 80, 3228 (1996).

${ }^{16}$ T. D. Moustakas, T. Lei, and R. J. Molnar, Physica B 185, 36 (1993).

${ }^{17}$ H. Riechert, R. Averbeck, A. Graber, M. Schienle, U. Strauss, and H. Tews, Mater. Res. Soc. Symp. Proc. 449, 149 (1997).

${ }^{18}$ L. T. Romano and T. H. Myers, Appl. Phys. Lett. 71, 3486 (1997).

${ }^{19}$ D. Kapolnek, S. Keller, R. Vetury, R. D. Underwood, P. Kozodoy, S. P. Den Baars, and U. K. Mishra, Appl. Phys. Lett. 71, 1204 (1997).

${ }^{20}$ E. C. Piquette, P. M. Bridger, Z. Z. Bandić, and T. C. McGill, Mater. Res. Soc. Symp. Proc. 512, 387 (1998).

${ }^{21}$ M. Richards-Babb, S. L. Buczkowski, Z. Yu, and T. H. Myers, Mater. Res. Soc. Symp. Proc. 395, 237 (1996).

${ }^{22}$ J. T. Kobayashi, N. P. Kobayashi, and P. D. Dapkus, J. Electron. Mater. 26, 1114 (1997). 